\title{
Colossal magneto-optical modulation at terahertz frequencies by counterpropagating femtosecond laser pulses in $\mathrm{Tb}_{3} \mathrm{Ga}_{5} \mathrm{O}_{12}$
}

\author{
Rostislav V. MikhaylovskiY, ${ }^{1,{ }^{*}}$ Ruslan R. Subkhangulov, ${ }^{1}$ TheO RASING $^{1}$, \\ ALEXEY V. KIMEL ${ }^{1,2}$ \\ ${ }^{1}$ Radboud Universit, Institute for Molecules and Materials, 6525 AJ Nijmegen, the Netherlands \\ ${ }^{2}$ Moscow State Technical University of Radio Engineering, Electronics and Automation, Moscow 119454, Russia \\ *Corresponding author: R.Mikhaylovskiy@science.ru.nl
}

Received XX Month XXXX; revised XX Month, XXXX; accepted XX Month XXXX; posted XX Month XXXX (Doc. ID XXXXX); published XX Month XXXX

\begin{abstract}
Single-frequency terahertz modulation of the magnetooptical Faraday effect with a record amplitude of the polarization rotation of $\sim 0.5^{\circ}$ is achieved using a slab of the etalon Faraday rotator crystal, $\mathrm{Tb}_{3} \mathrm{Ga}_{5} \mathrm{O}_{12}$. The modulation is the result of the interaction of two counterpropagating laser pulses via the optical Kerr effect. The frequency of the modulation is determined by the applied magnetic field and is continuously tunable in the terahertz frequency range between 0 and 0.7 THz. (c) 2016 Optical Society of America
\end{abstract}

OCIS codes: $\mathrm{xxxxx \times x \times xxx}$

http://dx.doi.org/xxxxxxxxxx

Magneto-optical effects embrace a collection of physical phenomena in which the polarization properties of light transmitted through or reflected from a magnetic material are modified due to the interaction with its magnetization [1]. In the case of the most common magneto-optical phenomenon, the Faraday effect, the light polarization rotates in the magnetic field applied to the material in the direction of light propagation [2]. The manipulation of light with magnetic fields has become commonplace in scientific research and technology, such as in optical isolators and modulators. However, the rapid development of the optical communications and optoelectronics calls for ever faster modification of optical properties of media and higher modulation frequencies.

Two main approaches for magneto-optical modulation are typically used. In the direct approach, the polarization of light is changed by the magnetic field varying in time $[3,4]$. The fastest magnetic fields are achieved for the freely propagating terahertz pulses, which however have broadband spectra [5]. A quasimonochromatic modulation at high (up to $\sim 1 \mathrm{THz}$ ) frequencies can be achieved by a second approach, where the polarization of light changes at the frequencies of electronic, lattice or spin resonances excited by femtosecond laser pulses [6] or ultrashort terahertz fields $[7,8]$.

Recently a fundamentally different strategy for the modulation of the Faraday rotation employing femtosecond laser pulses and the optical Kerr effect was demonstrated $[9,10]$. In the experiment, a magneto-optical crystal $\mathrm{Tb}_{3} \mathrm{Ga}_{5} \mathrm{O}_{12}$, subjected to a static magnetic field, was pumped by an intense femtosecond laser pulse, and subsequently probed by another weak pulse. In this way, it was demonstrated that the frequency of the magneto-optical modulation can be continuously tuned with the help of a magnetic field between zero and $1.1 \mathrm{THz}$.

Yet, while being the proof-of-principle demonstration of the feasibility of tunable terahertz Faraday modulation, the results of Ref. 9 left a lot of room for future developments. The demonstration exploited a pump pulse severely attenuated by the reflection, which resulted in a rather weak amplitude of the modulation of the order of $\sim 10$ mdeg.

In response to the aforementioned limitation, here we extend the concept by performing an experiment in which the pump pulse creating the Kerr dichroic inhomogeneity propagates against the modulated probe pulse from the very start. In this way we achieve a record value of the modulation as high as $0.5^{\circ}$. 


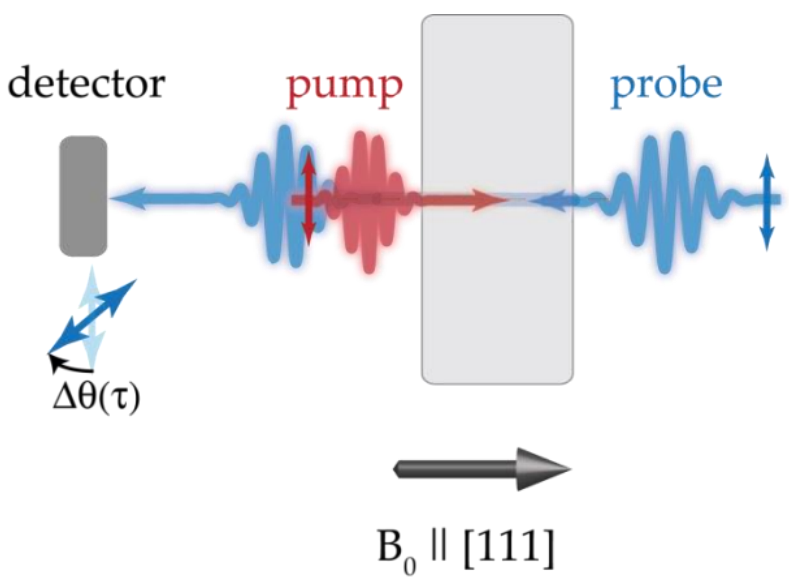

Fig 1. The geometry of the experiment. An intense femtosecond pump pulse $(\lambda=800 \mathrm{~nm}, h v=1.55 \mathrm{eV})$ is incident along the sample normal, which coincides with the [111] direction of the crystal. A weaker probe pulse $(\lambda=500 \mathrm{~nm}, h v=2.5 \mathrm{eV})$ falls onto the sample from the other side along the same direction. The rotation of the transmitted probe pulse $\Delta \theta(\tau)$ is measured by means of the optical balanced detector as a function of the time delay $\tau$ between the pump and probe. The sample is put in a constant magnetic field $\mathbf{B}_{0}$ applied along the [111] axis.

The measurements were conducted on a $10 \times 5 \times 1 \mathrm{~mm}^{3}$ single crystal plate of $\mathrm{Tb}_{3} \mathrm{Ga}_{5} \mathrm{O}_{12}$ cut such that the surface of the plate is a (111) plane. The $\mathrm{Tb}_{3} \mathrm{Ga}_{5} \mathrm{O}_{12}$ is a paramagnetic cubic crystal with excellent transparency in the visible range and the highest Faraday rotation that makes it a standard magneto-optical material for Faraday isolators [11]. In our experiment, schematically drawn in Fig. 1, the pump and probe laser pulses with duration $\sim 70 \mathrm{fs}$ fall on the sample from opposite sides, so that the beams are antiparallel inside the sample. The pump pulse has a central photon energy of $1.55 \mathrm{eV}$, while the photon energy of the probe pulse is $2.5 \mathrm{eV}$. Both beams are focused onto the sample with a spot diameter of $\sim 100$ $\mu \mathrm{m}$. The fluence of the probe pulse is two orders of magnitude smaller than that of the pump. By varying the difference between the lengths of the beampaths for the pump and probe pulses, we measured the temporal evolution of the pump-induced Faraday rotation of the probe.

The indicative Faraday signals are shown in Fig. 2 (a) for different strengths of the applied magnetic field. It is seen from the figure that each trace between 0 and $\sim 14$ ps is characterized by single-frequency oscillations. The period of the oscillations decreases with the field starting from a very long one at zero field. The Faraday modulation frequency as a function of the applied magnetic field is shown in Fig. 2 (b). It exhibits a fast linear increase for fields below $\sim 20 \mathrm{kG}$ and a slower growth for larger fields. Such behaviour follows the sample magnetization [12-14] and is similar to that observed in Ref. 9 for the Faraday modulation via the optical Kerr effect. Therefore we conclude that the oscillations in the present case have the same origin.

The strong electric field induces an optical anisotropy in any material by creating a dipole moment proportional to the square of the field $\left.{ }^{15}\right]$. The dipole is oriented along the electric field in a cubic medium. In this way the intense pump pulse creates the optical anisotropy axis along its polarization. The fast relaxation of the transient dipole moment results in the existence of the optical anisotropy only during the action of the pulse. The probe pulse experiences birefringence as it propagates through the region excited by pump. In the presence of absorption the probe pulse acquires rotation of its polarization. [16,17] This polarization rotation $\Delta \theta$ due to the optical Kerr effect is determined by the angle $\varphi$ between the polarization of the probe pulse and the optical anisotropy axis created by the pump as

$$
\Delta \theta \sim \sin 2 \varphi \text {. }
$$
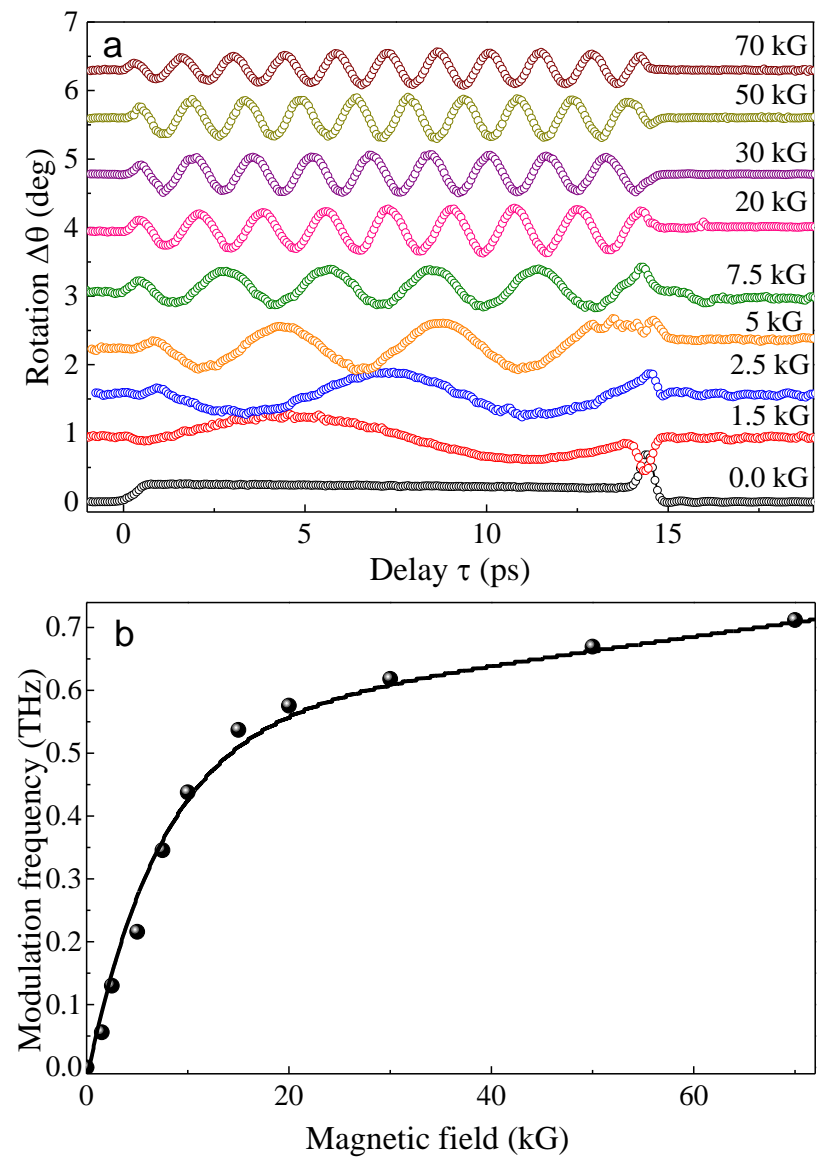

Fig 2. (a). Transient pump-induced Faraday rotation of the probe for different magnetic fields at temperature $T=1.9 \mathrm{~K}$. (b). The frequency of the Faraday modulation as a function of the applied field (spheres). The line follows the normalized magnetization of $\mathrm{Tb}_{3} \mathrm{Ga}_{5} \mathrm{O}_{12}$ as a function of the magnetic field applied along [111] axis. The magnetization curve was obtained by fitting the data from Ref. 12 with the exponential function.

This angle changes due to the static Faraday effect for both the pump and the probe pulses as

$$
\begin{aligned}
& \varphi= \\
& -\alpha_{\mathrm{pr}} M L+\left(\alpha_{\mathrm{pr}}+\alpha_{\mathrm{pu}}\right) M \frac{c \tau}{n_{\mathrm{pr}}+n_{\mathrm{pu}}},
\end{aligned}
$$

where $\alpha_{\mathrm{pu}}$ and $\alpha_{\mathrm{pr}}$ are the magneto-optical coefficients at the wavelength of the pump and probe, respectively; $M$ is the sample magnetization; $L$ is the sample thickness; $c$ is the speed of light in vacuum; $n_{\mathrm{pu}}$ and $n_{\mathrm{pr}}$ are the group refractive indices for the pump and probe, respectively. The time delay $\tau$ between the 
pulses is chosen so that at $\tau=0$ the pump and probe meet at the pump facet of the sample (left side of the sample in Fig. 1). Substituting Eq. (2) into Eq. (1) one can see that the Faraday rotation of the probe is an oscillatory function of the time delay $\tau$ with the frequency

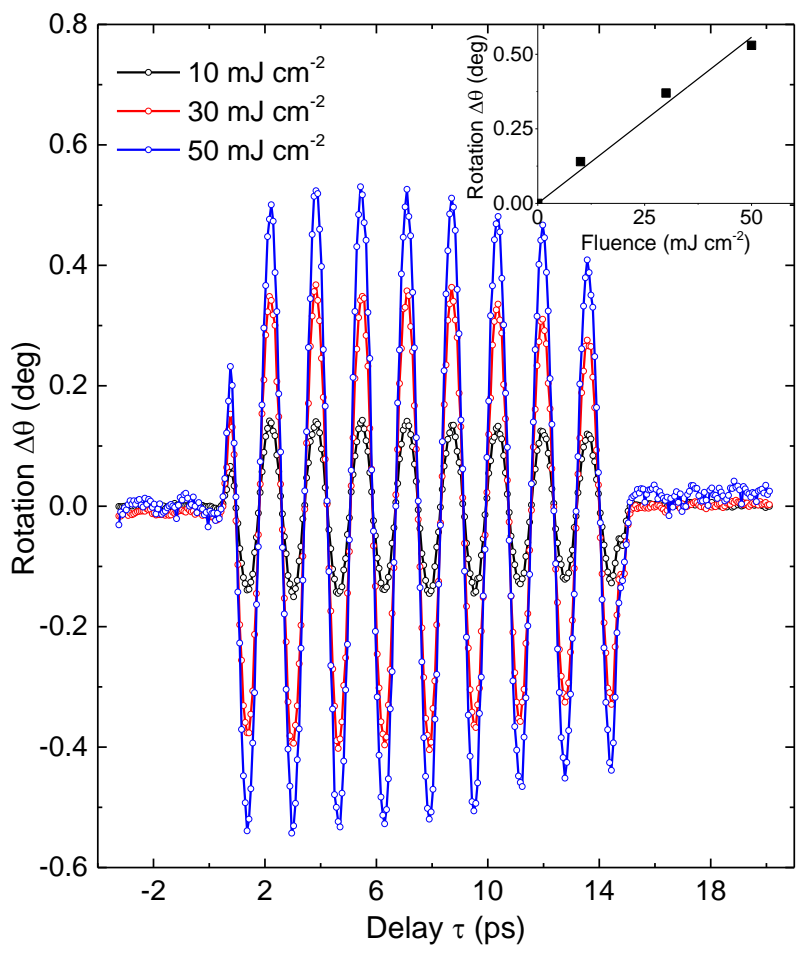

Fig 3. Transient Faraday modulation for different fluences of the pump pulse at temperature $T=1.9 \mathrm{~K}$. The inset shows the modulation amplitude as a function of the pump fluence (squares) together with a linear fit (solid line).

$$
\Omega=2 \frac{\alpha_{\mathrm{pr}}+\alpha_{\mathrm{pu}}}{n_{\mathrm{pr}}+n_{\mathrm{pu}}} c M,
$$

which is proportional to the sample magnetization. For fields below $20 \mathrm{kG}$ the magnetization is linearly proportional to the applied magnetic field so that $\Omega=2 \frac{V_{\mathrm{pr}}+V_{\mathrm{pu}}}{n_{\mathrm{pr}}+n_{\mathrm{pu}}} c B_{0}$, where $V_{\text {pu }}$ and $V_{\text {pr }}$ are the Verdet constants at the wavelength of the pump and probe, respectively. This equation matches the formula for the modulation frequency obtained in Ref. 9.

The probe polarization rotates due to the dichroic inhomogeneity induced by the counter-propagating pump pulse via the optical Kerr effect. The relative polarization angle is controlled by the position where the probe encounters the pump pulse inside the crystal. This position is set by the time delay between the pump and probe pulses. Therefore, the polarization of the probe oscillated as a function of the pump-probe delay, with the modulation frequency $\Omega$. However, the Kerr-induced signal can be only observed for time delays $0<\tau<\frac{n_{\mathrm{pr}}+n_{\mathrm{pu}}}{c} L$ when the pump and probe pulses can meet inside the sample. For our experiment we have $L=1 \mathrm{~mm}, n_{\mathrm{pu}}=1.953$ and $n_{\mathrm{pr}}=1.985$. Hence we estimate the duration of the modulation signal to be $\Delta \tau \approx 13 \mathrm{ps}$, a result that agrees well with the experimental data in Fig. 2 (a). Note that at zero field the optical pulses propagating through the crystal do not exhibit a Faraday rotation and therefore the angle given by Eq. (1) is constant inside the entire crystal. Therefore if no field is applied, no oscillations are observed and independently on the position in the crystal where the probe encounters the pump pulse, the optical Kerr effect results in the same polarization rotation.

Fig. 3 shows the Faraday modulation as a function of the pump fluence. The amplitude of the modulation increases linearly with the fluence while the modulation frequency does not change. Note the amplitude of the modulation reaches $\sim 0.5^{\circ}$, that is a record value of the Faraday rotation in pump-probe experiments, where typical signals are of the order of mdeg [18]. Such a large amplitude is achieved via a non-linear optical interaction between the pump and probe pulses. For the pulses propagating in the same direction this interaction results in a high Gaussian-like peak corresponding to the cross-correlation between their envelopes [19]. This peak at $\tau \approx 14 \mathrm{ps}$ is seen in some of our signals shown in Fig. 2 (a). It arises due to the interaction between the probe and the pump reflected from a surface of the sample and propagating in the same direction as the probe. When the pulses propagate against each other the Kerr interaction results in a boxcar-like signal which is periodically modulated due to the static Faraday rotation.

To conclude, we have demonstrated a manyfold enhancement of the Faraday modulation at $\mathrm{THz}$ frequencies. In our experiment the optical Kerr effect introduces an inhomogeneity in the magneto-optical medium and thus changes the total magnetooptical response. The oscillations are observed only in non-zero magnetic field that confirms that in the observed phenomenon the optical Kerr effect modulates the magneto-optical Faraday effect. The amplitude of the modulation is of the order of $0.5^{\circ}$. Our finding opens new possibilities for the design of all-optical magnetooptical devices, such as Faraday isolators, operating at THz clockrates.

Funding. the European Research Council ERC Grant agreement No. 257280 (Femtomagnetism) and No 62001706 (Exchange).

Acknowledgment. We thank A. Toonen for technical assistance, A. K. Zvezdin for fruitful discussions and V. V. Kruglyak for providing the sample.

\section{References}

1. A. K. Zvezdin and A. V. Kotov, Modern Magneto-optics and Magnetooptical Materials (IOP, Bristol, 1997).

2. M. Faraday. Phil. Trans. R. Soc. 136, 1 (1846).

3. J. A. Riordan, F. G. Sun, Z. G. Lu, and X.-C. Zhang, Appl. Phys. Lett. 71, 1452 (1997). 
4. N. Kumar, Kumar, A. Strikwerda, K. Fan, .X. Zhang, R. D. Averitt, P. Planken, and A. Adam, Opt. Express 20, 11277 (2012).

5. T. Kampfrath, K. Tanaka, and K. A. Nelson, Nature Photon. 7, 680 (2013).

6. A. Kirilyuk, A. V. Kimel, and Th. Rasing, Rev. Mod. Phys. 82, 2731 (2010).

7. T. Kampfrath, A. Sell, G. Klatt, A. Pashkin, S. Mährlein, T. Dekorsy, M. Wolf, M. Fiebig, A. Leitenstorfer and R. Huber, Nature Photon. 5, 31 (2011).

8. C. Vicario, C. Ruchert, F. Ardana-Lamas, P. M. Derlet, B. Tudu, J. Luning and C. P. Hauri. Nature Photon. 7, 720 (2013).

9. R. R. Subkhangulov, R. V. Mikhaylovskiy, A. K. Zvezdin, V. V. Kruglyak, Th. Rasing and A. V. Kimel, Nature Photon. 10, 111 (2016). 10. B. G. Ghamsari and P. Berini, Nature Photon. 10, 74 (2016).

11. E. A. Mironov, D. S. Zheleznov, A. V. Starobor, A. V. Voitovich, O. V. Palashov, A. M. Bulkanov, and A. G. Demidenko, Opt. Lett. 40, 2794 (2015).

12. M. Guillot, and A. Marchand, J. Phys. C 18, 3547 (1985).

13. K. Kamazawa, D. Louca, R. Morinaga, T. J. Sato, Q. Huang, J. R. D. Copley, and Y. Qiu, Phys. Rev. B 78, 064412 (2008).

14. U. Löw, S. Zvyagin, M. Ozerov, U. Schaufuss, V. Kataev, B. Wolf, B. Lüthiet. Eur. Phys. J. B 86, 87 (2013).

15. R. Righini, Science, 262, 1386 (1993).

16. S. V. Popov, Y. P. Svirko, and N. I. Zheludev, J. Opt. Soc. Am. B 13, 2729 (1996).

17. Y. P. Svirko and N. I. Zheludev, Polarization in the Nonlinear Optics (John Wiley \& Sons, 1999).

18. A. Kalashnikova, A. V. Kimel and R. V. Pisarev, Physics-Uspekhi 58, 969 (2015).

19. R. V. Mikhaylovskiy, E. Hendry, V. V. Kruglyak. Phys. Rev. B 86, 100405 (2012). 
[1]. A. K. Zvezdin and A. V. Kotov, Modern Magneto-optics and Magnetooptical Materials (IOP, Bristol, 1997).

[2]. M. Faraday. On the magnetization of light and the illumination of magnetic lines of force. Phil. Trans. R. Soc. 136, 1 (1846).

[3]. J. A. Riordan, F. G. Sun, Z. G. Lu, and X.-C. Zhang. Free-space transient magneto-optic sampling. Appl. Phys. Lett. 71, 1452 (1997). [4]. N. Kumar, Kumar, A. Strikwerda, K. Fan, .X. Zhang, R. D. Averitt, P. Planken, and A. Adam. THz near-field Faraday imaging in hybrid metamaterials. Opt. Express 20, 11277 (2012).

[5]. T. Kampfrath, K. Tanaka, and K. A. Nelson. Resonant and nonresonant control over matter and light by intense terahertz transients. Nature Photon. 7, 680 (2013).

[6]. A. Kirilyuk, A. V. Kimel, and Th. Rasing. Ultrafast optical manipulation of magnetic order. Rev. Mod. Phys. 82, 2731 (2010).

[7]. T. Kampfrath, A. Sell, G. Klatt, A. Pashkin, S. Mährlein, T. Dekorsy, M. Wolf, M. Fiebig, A. Leitenstorfer and R. Huber. Coherent terahertz control of antiferromagnetic spin waves. Nature Photon. 5, 31 (2011). [8]. C. Vicario, C. Ruchert, F. Ardana-Lamas, P. M. Derlet, B. Tudu, J. Luning and C. P. Hauri. Off-resonant magnetization dynamics phaselocked to an intense phase-stable terahertz transient. Nature Photon. 7, 720 (2013).

[9]. R. R. Subkhangulov, R. V. Mikhaylovskiy, A. K. Zvezdin, V. V. Kruglyak, Th. Rasing and A. V. Kimel. Terahertz modulation of the Faraday rotation by laser pulses via the optical Kerr effect. Nature Photon. 10, 111 (2016).

[10]. B. G. Ghamsari and P. Berini. Fundamental optical physics: Nonlinear optics rules magnetism. Nature Photon. 10, 74 (2016).

[11]. E. A. Mironov, D. S. Zheleznov, A. V. Starobor, A. V. Voitovich, O. V. Palashov, A. M. Bulkanov, and A. G. Demidenko. Large-aperture Faraday isolator based on a terbium gallium garnet crystal. Opt. Lett. 40, 2794 (2015).

[12]. M. Guillot, and A. Marchand. Step-like magnetisation curves in $\mathrm{Tb}_{3} \mathrm{Ga}_{5} \mathrm{O}_{12}$. J. Phys. C 18, 3547 (1985).

[13]. K. Kamazawa, D. Louca, R. Morinaga, T. J. Sato, Q. Huang, J. R. D. Copley, and Y. Qiu. Field-induced antiferromagnetism and competition in the metamagnetic state of terbium gallium garnet. Phys. Rev. B 78, 064412 (2008).

[14]. U. Löw, S. Zvyagin, M. Ozerov, U. Schaufuss, V. Kataev, B. Wolf, B. Lüthiet. Magnetization, magnetic susceptibility and ESR in $\mathrm{Tb}_{3} \mathrm{Ga}_{5} \mathrm{O}_{12}$. Eur. Phys. J. B 86, 87 (2013).

[15]. R. Righini. Ultrafast optical Kerr effect in liquids and solids. Science, 262, 1386 (1993).

[16]. S. V. Popov, Y. P. Svirko, and N. I. Zheludev. Pump-probe reflective polarization-sensitive nonlinear optics. J. Opt. Soc. Am. B 13, 2729 (1996).

[17]. Y. P. Svirko and N. I. Zheludev, Polarization in the Nonlinear Optics (John Wiley \& Sons, 1999).

[18]. A. Kalashnikova, A. V. Kimel and R. V. Pisarev. Ultrafast optomagnetism. Physics-Uspekhi 58, 969 (2015).

[19]. R. V. Mikhaylovskiy, E. Hendry, V. V. Kruglyak. Ultrafast inverse Faraday effect in a paramagnetic terbium gallium garnet crystal. Phys. Rev. B 86, 100405 (2012). 